\title{
Summary of Super Doubler Approach on Exact Lattice Supersymmetry
}

\author{
Alessandro D'Adda \\ INFN Sezione di Torino, and \\ Dipartimento di Fisica Teorica, Universita di Torino \\ I-10125 Torino, Italy \\ E-mail: dadda@to.infn.it \\ Noboru Kawamoto* \\ Department of Physics, Hokkaido University \\ Sapporo, 060-0810 Japan \\ E-mail: kawamoto@particle.sci.hokudai.ac.jp
}

\section{Jun Saito}

Division of Natural Science Department of Human Science

Obihiro University of Agriculture and Veterinary Medicine

Obihiro, Japan

E-mail: jsaito@obihiro.ac.jp

\begin{abstract}
We have proposed a lattice SUSY formulation which we may call super doubler approach, where chiral fermion species doublers and their bosonic counter parts are either identified as super partners or truncated by chiral conditions. We claim that the super symmetry is exactly kept on the lattice. However the formulation is nonlocal and breaks lattice translational invariance. We argue that these features cause no fundamental difficulties in the continuum limit. Although a naive version of this formulation breaks associativity of the product of fields we have found a modified super doubler approach that recovers the associativity and is applicable to super Yang-Mills theory. It turns out that this formulation is essentially equivalent to the continuum formulation and thus keeps all the symmetry exact even at a finite lattice constant. Inspired by this formulation we propose a non-local lattice field theory formulation which is free of chiral fermion problem and has the same exact lattice symmetry as continuum theory.
\end{abstract}

The 33rd International Symposium on Lattice Field Theory

14 - 18 July 2015

Kobe International Conference Center, Kobe, Japan*

\footnotetext{
* Speaker.
} 


\section{Introduction}

Although realization of exact SUSY on the lattice has a long history it is uncompleted subject[1]. We hope to find the clue for a new field theory formulation by understanding the fundamental difficulties to realize exact lattice SUSY. A part of the exact lattice SUSY of extended algebra was realized and extensively investigated[2] 3]. The link approach of super Yang-Mills theory was also proposed[4]. It turned out this formulation needs to be non-commutative and has Hopf algebraic symmetry [5].

There are two major difficulties to realize exact supersymmetry on the lattice:

1) Lattice counter part of differential operator, a difference operator, in the SUSY algebra does not satisfy Leibniz rule which causes non-vanishing nature of surface terms.

2) Naive fermion formulation of chiral fermion generates fermion species doublers which breaks the balance of boson and fermion number. Any other fermion formulation may generate obstacles for realization of exact lattice SUSY due to the different treatment of fermions and bosons.

Within the framework of locality, lattice translational invariance, and associativity, it was claimed that Leibniz rule of difference operator cannot be realized[6. One may thus need to give up some of these principles to realize exact lattice SUSY. In fact it was pointed out that SLAC derivative would be the only solution compatible with the lattice SUSY version of Ginsparg-Wilson relation[7]. It is well recognized that SLAC derivative is nonlocal derivative. It has also been pointed out that this type of nonlocality does not cause fundamental problem for the realization of SUSY on the lattice 8, 9 Here we investigate a formulation to accept nonlocality but keep "exact SUSY" on the lattice.

In order to avoid chiral fermion species doublers we may introduce Wilson term and ask if one can obtain lattice SUSY invariance perturbatively. It was noticed that bosons also need corresponding terms to the Wilson terms of fermions 10 11. Modern resolution of chiral fermion problem for QCD may give a suggestion that Ginsparg-Wilson fermion would solve lattice SUSY fermion treatment. It was pointed out that Majorana condition has a difficulty of compatibility with Ginsparg-WiIson relation[12]. After all in these formulations fermions are formulated differently from bosons the realization of exact lattice SUSY is difficult.

Here we investigate a formulation of exact lattice SUSY invariant formulation within a framework of nonlocal field theory.

\section{Modified lattice momentum conservation}

In order to realize lattice SUSY invariance it was suggested in the first pioneering paper by Dondi and Nicolai[1] that lattice momentum conservation could be modified as follows:

$$
\delta\left(p_{1}+p_{2}+\cdots\right) \longrightarrow \delta\left(\frac{\sin a p_{1}}{a}+\frac{\sin a p_{2}}{a}+\cdots\right)
$$

where $\frac{\sin a p_{\mu}}{a}$ is the momentum representation of symmetric difference operator. We claim that this replacement is not enough to solve the second species doubler problem 2) mentioned the above.

Let us consider what the meaning of this replacement is. We usually identify the lattice momentum $-\frac{\pi}{a} \leq p_{\mu} \leq \frac{\pi}{a}$ as an angular variable identified from the momentum representation 
of difference operator $\frac{\sin a p_{\mu}}{a}$. This lattice momentum approaches to the continuum momentum $-\infty<\hat{p}_{\mu}<\infty$ in the continuum limit $a \rightarrow 0$. Since the lattice momentum is an angular variable it has different topological nature from the continuum momentum, which is the origin of chiral fermion species doubling. The most important advantage of this identification is that discrete lattice translation invariance is kept with this choice. We can, however, argue later that lattice translational invariance is not the mandatory requirement for the recovery of Poincare invariance in the continuum limit. Then we can equally well identify a lattice momentum $\Delta\left(p_{\mu}\right)$ as an corresponding lattice momentum if $\Delta\left(p_{\mu}\right) \rightarrow p_{\mu}(a \rightarrow 0)$.

In contrast to the choice of $\Delta(p)=\frac{\sin a p}{a}$, what could be the best choice to solve the second problem 2) at the same time ? Coordinate representation of this symmetric difference operator which is Hermitian has the following well known form:

$$
i \Delta_{s} f(x)=i \frac{f(x+a)-f(x-a)}{2 a} \rightarrow \frac{\sin a p}{a} \tilde{f}(p) .
$$

If we identify this as the translation generator the minimal unit of translation should be identified as two lattice unit. This point of view can be also understood by recognizing that species doubler state is a real physical state. We may define a new momentum for species doubler as $\frac{\sin a\left(\frac{\pi}{a}-p^{\prime}\right)}{a} \sim$ $p^{\prime}(a \rightarrow 0)$. The coordinate representation of the corresponding field is $\tilde{\psi}\left(\frac{\pi}{a}-p^{\prime}\right) \rightarrow(-1)^{\frac{x}{a}} \psi(-x)$ where $x=n a(n \in Z)$ is lattice coordinate. Translational invariant species doubler state is thus $(-1)^{n}$ const. which needs two lattice unit for translation invariance.

The above observation suggests us a picture that we should construct a lattice formulation which leads single lattice as a minimal translation. In other words we need to introduce half lattice coordinates. Then what is the half lattice translation generator? A half lattice translation can be nicely understood as supersymmetry translation, which is compatible with the following simplest SUSY algebra: $\{Q, Q\}=2 P=2 i \Delta_{s}$, where $i \Delta_{s}=\frac{2}{a} \sin \frac{p a}{2}$ is the momentum representation of single lattice translation generator while $Q$ is the half lattice translation generator. Our first step proposal for $\Delta(p)$ is

$$
\Delta(p)=\frac{2}{a} \sin \frac{p a}{2}
$$

We claim that this choice of $\Delta(p)$ solves two lattice SUSY difficulties 1) and 2) at the same time for non-gauge SUSY models where the following replacement need to be introduced:

$$
\delta\left(p_{1}+p_{2}+\cdots\right) \longrightarrow \delta\left(\Delta\left(p_{1}\right)+\Delta\left(p_{2}\right)+\cdots\right) .
$$

In fact we have found momentum representation of lattice SUSY algebra for $\mathrm{D}=1,2$ and $\mathrm{N}=2$ Wess-Zumino models. In one dimension $(\mathrm{D}=1)$ we introduce bosonic and fermionic composite fields $\Phi(p)$ and $\Psi(p)$ each of which includes original field and species doubler fields for both bosonic and fermionic composite fields. SUSY transformation of the composite fields are

$$
\begin{aligned}
& Q_{1} \Phi(p)=i \cos \frac{a p}{4} \Psi(p), \quad Q_{1} \Psi(p)=-4 i \sin \frac{a p}{4} \Phi(p), \\
& Q_{2} \Phi(p)=\cos \frac{a p}{4} \Psi\left(\frac{2 \pi}{a}-p\right), \quad Q_{2} \Psi\left(\frac{2 \pi}{a}-p\right)=4 \sin \frac{a p}{4} \Phi(p),
\end{aligned}
$$

which satisfy the following $\mathrm{N}=2$ twisted SUSY algebra:

$$
Q_{1}^{2}=Q_{2}^{2}=2 \sin \frac{a p}{2}, \quad\left\{Q_{1}, Q_{2}\right\}=0,
$$


where the dimensionless supercharges are introduced. We found the following action which is invariant under the above lattice SUSY algebra[13]:

$$
\begin{aligned}
S^{(n)} \quad & =g_{0}^{(n)} a^{n} \frac{4}{n !} \int_{-\frac{\pi}{a}}^{\frac{3 \pi}{a}} \frac{d p_{1}}{2 \pi} \cdots \frac{d p_{n}}{2 \pi} 2 \pi \delta\left(\sum_{i=1}^{n} \sin \frac{a p_{i}}{2}\right) \\
\times & G\left(p_{1}, p_{2}, \cdots, p_{n}\right)\left[2 \sin ^{2} \frac{a p_{1}}{4} \Phi\left(p_{1}\right) \Phi\left(p_{2}\right) \cdots \Phi\left(p_{n}\right)+\right. \\
+ & \left.\frac{n-1}{4} \sin \frac{a\left(p_{1}-p_{2}\right)}{4} \Psi\left(p_{1}\right) \Psi\left(p_{2}\right) \Phi\left(p_{3}\right) \cdots \Phi\left(p_{n}\right)\right],
\end{aligned}
$$

where dimensionless $\Delta\left(p_{i}\right)=\sin \frac{a p_{i}}{2}$ is chosen as conserved lattice momentum of (2.4) in this expression. For $n=2$ this action gives kinetic and mass terms of one dimensional $\mathrm{N}=2$ Wess-Zumino action. Here we identify the species doublers as super partners for boson and fermion:

$$
\begin{aligned}
& \Phi(p)=a^{-\frac{3}{2}} \varphi(p), \Psi(p)=a^{-1} \psi_{1}(p), \\
& \Psi\left(\frac{2 \pi}{a}-p\right)=i a^{-1} \psi_{2}(p), \Phi\left(\frac{2 \pi}{a}-p\right)=-\frac{a^{-\frac{1}{2}}}{4} D(p) .
\end{aligned}
$$

Similarly for $\mathrm{D}=\mathrm{N}=2$ lattice SUSY the kinetic terms of Wess-Zumino action can be found as:

$$
\begin{aligned}
S_{K}= & 4 \int_{-\frac{\pi}{a}}^{\frac{\pi}{a}} d p_{+} d p_{-} d q_{+} d q_{-} \delta\left(p_{+}+q_{+}\right) \delta\left(p_{-}+q_{-}\right)\left[-4 \bar{\Phi}(p) \sin \frac{a q_{+}}{2} \sin \frac{a q_{-}}{2} \Phi(q)\right. \\
& \left.-\bar{F}(p) F(q)+2 \bar{\Psi}_{2}(p) \sin \frac{a q_{+}}{2} \Psi_{2}(q)+2 \bar{\Psi}_{1}(p) \sin \frac{a q_{-}}{2} \Psi_{1}(q)\right]
\end{aligned}
$$

where for the kinetic term the proposed momentum conservation coincide with the standard lattice momentum conservation. The interaction terms for the Wess-Zumino action can be given by

$$
S_{n}=\int \prod_{j=1}^{n} d^{2} p_{j} V_{n}(p) n\left[i F\left(p_{1}\right) \prod_{j=2}^{n} \Phi\left(p_{j}\right)+(n-1) \Psi_{2}\left(p_{1}\right) \Psi_{1}\left(p_{2}\right) \prod_{j=3}^{n} \Phi\left(p_{j}\right)\right]+\text { h.c. }
$$

where $V_{n}(p)$ includes sine momentum conservation:

$$
V_{n}(p)=a^{2 n} g_{n} \delta^{(2)}\left(\sin \frac{a p_{1}}{2}+\sin \frac{a p_{2}}{2}+\cdots+\sin \frac{a p_{n}}{2}\right) .
$$

Chiral representation of $\mathrm{D}=\mathrm{N}=2$ SUSY algebra for the Wess-Zumino model can be fully derived with momentum representation of difference operator as momentum generator. See the details in [14].

Chiral conditions impose the following relations for composite field of bosons and fermions $\Phi_{A}$, each of which include 4 species doublers:

$$
\Phi_{A}\left(p_{+}, p_{-}\right)=\Phi_{A}\left(\frac{2 \pi}{a}-p_{+}, p_{-}\right)=\Phi_{A}\left(p_{+}, \frac{2 \pi}{a}-p_{-}\right)=\Phi_{A}\left(\frac{2 \pi}{a}-p_{+}, \frac{2 \pi}{a}-p_{-}\right) .
$$

For other composite fields $F(p), \Psi_{i}(p)$ similar chiral conditions are needed. The anti-chiral fields carrying bar need similar relations. In this way four species doubler degrees of freedom is all truncated by the chiral conditions. It is interesting to note that the coordinate representation of the first equality in eq. 2.12) is given as:

$$
\Phi_{A}\left(x_{+}, x_{-}\right)=(-1)^{\frac{2 x_{+}}{a}} \Phi_{A}\left(-x_{+}, x_{-}\right),
$$


where the second and third equality can be given similarly. This relation shows that we have introduced twice larger degrees of freedom by introducing half lattice structure but it is truncated by half by the chiral condition for each dimension. It is thus sufficient to consider positive lattice coordinate for each dimensional direction.

Since the species doubler degrees are killed now we can identify each composite fields as component fields:

$$
a^{2} \Phi(p) \rightarrow \varphi(p), \quad a^{\frac{3}{2}} \Psi_{i}(p) \rightarrow \psi_{i}(p), \quad a F(p) \rightarrow f(p) .
$$

In this way for the treatment of species doubler degrees of freedom there are two possible ways: A) Identify the species doubler fields as super partners as in one dimensional treatment in eq. 2.9. B) Kill the all the species doubler degrees of freedom by chiral conditions as in two dimensional treatment in eq.(2.12).

\section{Recovery of Leibniz rule and associativity}

We claim that the actions given above satisfy $\mathrm{N}=2$ exact lattice SUSY algebra in one and two dimensions. How do we understand that the problem of difference operator not satisfying Leibniz rule can be realized in the coordinate space ? Convolution of two product fields in the momentum space corresponds to the normal product in the coordinate space:

$$
(F \cdot G)(p)=\int d^{2} p_{1} d^{2} p_{2} F\left(p_{1}\right) G\left(p_{2}\right) \delta^{(2)}\left(p-p_{1}-p_{2}\right) \longrightarrow(F \cdot G)(x)=F(x) G(x) .
$$

Correspondingly the change of the momentum conservation into sine of the momentum leads to define a new product:

$$
(F * G)(p)=\int d^{2} p_{1} d^{2} p_{2} F\left(p_{1}\right) G\left(p_{2}\right) \delta^{(2)}\left(\Delta(p)-\Delta\left(p_{1}\right)-\Delta\left(p_{2}\right)\right) \longrightarrow(F * G)(x)=F(x) * G(x) .
$$

If we now operate the momentum representation of difference operator to the product we naturally lead Leibniz rule in the momentum representation due to the new momentum conservation:

$\Delta(p)(F * G)(p)=\int d^{2} \hat{p}_{1} d^{2} \hat{p}_{2}\left[\Delta\left(p_{1}\right) F\left(p_{1}\right) G\left(p_{2}\right)+F\left(p_{1}\right) \Delta\left(p_{2}\right) G\left(p_{2}\right)\right] \delta^{(2)}\left(\Delta(p)-\Delta\left(p_{1}\right)-\Delta\left(p_{2}\right)\right.$.

The coordinate representation of this expression tells us that difference operator $\hat{\partial}_{x}$ satisfy the Libniz rule on the new $*$-product:

$$
\hat{\partial}_{x}(F * G)(x)=\left(\hat{\partial}_{x} F(x)\right) * G(x)+F(x)\left(\hat{\partial}_{x} * G(x)\right) .
$$

Unfortunately this new $*$-product is unavoidably nonlocal[6]. The explicit expression of the $*$-product in the coordinate space can be found in [13, 14].

It turns out, however, that this $*$-product is not associative due to the limited range of $|\Delta(p)|$.

$$
\left(\left(\Phi_{3} * \Phi_{2}\right) * \Phi_{1}\right)(p) \neq\left(\Phi_{3} *\left(\Phi_{2} * \Phi_{1}\right)\right)(p)
$$

In order to formulate gauge theory associativity is crucial since gauge transformation is non-linear, which can be seen from the following manipulation proving gauge invariance:

$$
\Phi^{\dagger}(x) * \Phi(x) \rightarrow\left(\Phi^{\dagger}(x) * e^{-\alpha(x)}\right) *\left(e^{\alpha(x)} \Phi(x)\right) \neq \Phi^{\dagger}(x) *\left(e^{-\alpha(x)} e^{\alpha(x)}\right) \Phi(x)=\Phi^{\dagger}(x) * \Phi(x) .
$$


We have, however, found an interesting solution of the choice for $\Delta(p)=\Delta_{G}(p)$ which satisfies associativity:

$$
\Delta_{G}(p)=\frac{1}{a} \log \frac{1+\sin \frac{a p}{2}}{1-\sin \frac{a p}{2}}
$$

where $\Delta_{G}\left( \pm \frac{\pi}{a}\right)= \pm \infty$. The coordinate representation of this differential operator $\Delta_{G}$ has the following nice but non-local form:

$$
\Delta_{G} \Phi(x)=\frac{2}{a} \sum_{k=1}^{\infty} \frac{(-1)^{k+1}}{2 k-1}\left[\Phi\left(x+\frac{(2 k-1) a}{2}\right)-\Phi\left(x-\frac{(2 k-1) a}{2}\right)\right]
$$

Replacing the $\Delta(p)$ in eq. (3.2) by $\Delta_{G}(p)$ we can define yet new $\star$-product which satisfies associativity:

$$
\left(\left(\Phi_{3} \star \Phi_{2}\right) \star \Phi_{1}\right)(p)=\left(\Phi_{3} \star\left(\Phi_{2} \star \Phi_{1}\right)\right)(p) .
$$

By the use of this formulation in principle we can formulate super Yang-Mills theory since lattice SUSY and gauge invariance could be assured.

Since the lattice momentum conservation is changed as in eq. (2.4) discrete lattice translational invariance is lost. However we claim that the invariance of the action for Poincare invariance can be assured by the corresponding invariance by

$$
\delta_{\varepsilon} \Phi_{A}(p)=-i \varepsilon \Delta_{G}(p) \Phi_{A}(p),
$$

where newly defined $\Delta_{G}(p)$ can be identified as a translation generator.

\section{Proposal of a new lattice field theory formulation}

Based on the formulation of employing $\Delta_{G}(p)$ as a differential operator we propose to define a new lattice field theory formulation which is equivalent to continuum theory of paying price of non-locality complication:

1) Introduce a half lattice structure. 2) Go to the momentum representation of a continuum formulation and replace all derivative operators by $\Delta_{G}(p)$ and the momentum conservation in (2.4) with $\Delta(p)=\Delta_{G}(p)$. 3) Kill the species doublers degrees of freedom by the equations as in (2.12). In the coordinate representation consider only the first quadrant of lattice space and replace all the product by $\star-$ product.

In this way we can construct non-local lattice field theory which has exact lattice symmetries of continuum theory and no chiral fermion problem. In fact this non-local lattice theory is equivalent to the continuum theory.

\section{Conclusion and Discussions}

We have proposed a lattice SUSY formulation which has the exact lattice SUSY especially for $\mathrm{D}=1,2$ and $\mathrm{N}=2$ Wess-Zumino models. Even though associativity is broken in these cases exact lattice SUSY is kept since SUSY transformation is linear in fields. Non-associativity does not sacrifice exact lattice SUSY for non-gauge cases. In fact exact SUSY has been confirmed even at the quantum level[15]. We have proposed a new lattice SUSY formulation by the use of $\Delta_{G}$ as a 
differential operator. It has the same exact lattice symmetries with continuum theory. In fact the formulation is non-local lattice field theory and equivalent to the continuum theory. In a sense this formulation presents "perfect action" [16] of lattice SUSY. One may wonder if this lattice theory is regularized or not. We consider that the lattice SUSY formulated by $\Delta_{G}$ is not regularized even though it is a lattice theory with a lattice constant introduced. This is a puzzling situation. If the non-local nature of the formulation is serious the formulation may be useless. We can,however, show that the locality of interactions is recovered in the continuum limit for both $\Delta(p)=\sin \frac{a p}{2}$ and $\Delta_{G}(p)$ formulations. The details will be given elsewhere [17].

\section{Acknowledgements}

We thank P.H.Damgaard for useful discussions and comments. This work was supported in part by Japanese Ministry of Education, Science, Sports and Culture under the grant number 22540261 and also by the research funds of Instituto Nazionale di Fisica Nucleare (INFN).

\section{References}

[1] P. H. Dondi and H. Nicolai, Nuovo Cim. A 41, 1 (1977).

[2] D. B. Kaplan, Nucl. Phys. Proc. Suppl. 129 (2004) 109.

[3] P. H. Damgaard and S. Matsuura, JHEP 0709 (2007) 097. Phys. Lett. B 661 (2008) 52.

[4] A. D’Adda, I. Kanamori, N. Kawamoto and K. Nagata, Nucl. Phys. Proc. Suppl. 140, 754 (2005), A. D'Adda, I. Kanamori, N. Kawamoto and K. Nagata, Phys. Lett. B 633, 645 (2006),

A. D'Adda, I. Kanamori, N. Kawamoto and K. Nagata, Nucl. Phys. B 798, 168 (2008).

[5] A. D’Adda, N. Kawamoto and J. Saito, Phys. Rev. D81 (2010) 065001.

[6] M. Kato, M. Sakamoto and H. So, JHEP 0805 (2008) 057.

[7] G. Bergner, F. Bruckmann, and J. M. Pawlowski, Phys. Rev. D79 (2009) 115007.

[8] G. Bergner, JHEP 1001 (2010) 024.

[9] D. Kadoh and H. Suzuki, Phys. Lett. B 684 (2010) 167.

[10] J. Bartels ad G. Kramer, Z.Phys. C20 (1983) 159.

[11] J. Giedt, R. Koniuk, E. Poppitz and T. Yavin, JHEP 12 (2004) 033.

[12] K.Fujikawa and M.Ishibashi, Phys. Let. B528 (2002) 295.

[13] A.D'Adda, A.Feo, I.Kanamori, N.Kawamoto and J.Saito, JHEP 1009 (2010) 059.

[14] A.D'Adda, I.Kanamori, N.Kawamoto and J.Saito, JHEP 1203 (2012) 043.

[15] Asaka K., D'adda A., Kawamoto N., Kondo Y., Nat. Acad. of Sci. Kazakhstan report Phys. and Math., No2, 288, March-April 2013,P.18 [arXiv:1302.1268[hep-lat]]

[16] P.Hasenfratz and F.Niedermayer, Nucl. Phys. B414 (1994) 785.

[17] A.D'Adda, N.Kawamoto and J.Saito, to appear. 